\title{
$K$-user Interference Channels: General Outer Bound and Sum-capacity for Certain Gaussian Channels
}

\author{
Daniela Tuninetti \\ Department of Electrical and Computer Engineering, \\ University of Illinois at Chicago, Illinois 60607, USA, \\ Email: danielat@uic.edu
}

\begin{abstract}
This paper derives an outer bound on the capacity region of a general memoryless interference channel with an arbitrary number of users. The derivation follows from a generalization of the techniques developed by Kramer and by Etkin et al for the Gaussian two-user channel. The derived bound is the first known outer bound valid for any memoryless channel.

In Gaussian noise, classes of channels for which the proposed bound gives the sum-rate capacity are identified, including degraded channels and a class of Z-channels.

Index Terms-Interference channel; Degraded channel; Outer bound; Sum-capacity; Z-channel;
\end{abstract}

\section{INTRODUCTION}

Determining the ultimate capacity limits of the general memoryless $K$-user InterFerence Channel ( $K$-IFC) is an open problem since its inception. The network considered in this work is depicted in Fig. 1 it consists of $K$ pairs of nodes and is defined by input alphabets $\left(\mathcal{X}_{1}, \ldots, \mathcal{X}_{K}\right)$, output alphabets $\left(\mathcal{Y}_{1}, \ldots, \mathcal{Y}_{K}\right)$, and a channel transition probability $P_{Y_{1}, \ldots, Y_{K} \mid X_{1}, \ldots, X_{K}}$. The only assumption on the channel is that it is memoryless. Source $i, i \in[1: K]$, wishes to communicate to destination $i$ an independent message $W_{i}$. A $\left(\mathrm{e}^{n R_{1}}, \ldots, \mathrm{e}^{n R_{K}}, n, \epsilon_{n}\right)$ code consists of $K$ encoding functions $\left[1: \mathrm{e}^{n R_{i}}\right] \rightarrow \mathcal{X}_{i}^{n}, K$ decoding functions $\widehat{W}_{i}: \mathcal{Y}_{i}^{n} \rightarrow$ $\left[1: \mathrm{e}^{n R_{i}}\right]$, such that $\operatorname{Pr}\left[\widehat{W}_{i}\left(Y_{i}^{n}\right) \neq W_{i}\right] \leq \epsilon_{n}, i \in[1: K]$. A rate-tuplet $\left(R_{1}, \ldots, R_{K}\right)$ is achievable if there exists a family of $\left(\mathrm{e}^{n R_{1}}, \ldots, \mathrm{e}^{n R_{K}}, n, \epsilon_{n}\right)$ codes such that $\epsilon_{n} \rightarrow 0$ as $n \rightarrow \infty$. The capacity region is the convex closure of the set of achievable rate-tuplet $\left(R_{1}, \ldots, R_{K}\right)$. The capacity is not known in general.

The goal of this paper is to derive an outer bound for the $K$-IFC that holds for any memoryless channel (not necessarily Gaussian) and for any $K \geq 2$.

\section{A. Past Work}

The capacity region of the 2-IFC is known if the interference is strong [1]-[3], if the channel outputs are deterministic and invertible functions of the inputs [4], and if the channel has a special form of degradeness [5], [6]. The largest known inner bound is due to Han and Kobayashi (HK) [7] and uses rate splitting and joint decoding. General outer bounds are due to Sato [1], [8], and Carleial [9] (see also Kramer [10, Th.5]).

For the Gaussian 2-IFC, the capacity region is fully known in strong interference only [3], [11], [12]. The sum-capacity is however known in mixed interference [13], [14], for the Zchannel [15], and in very weak interference [14], [16], [17]. In

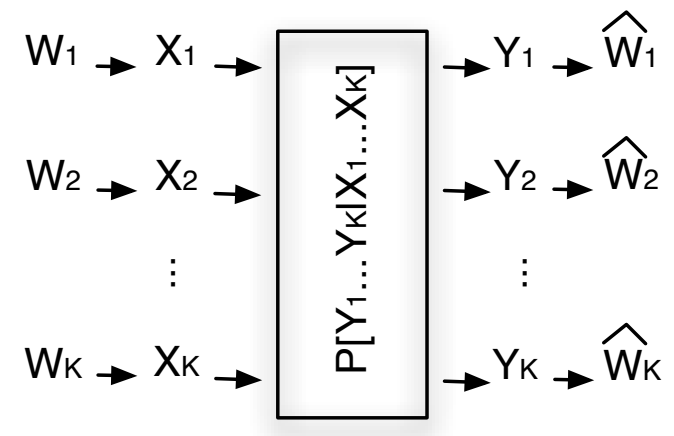

Fig. 1. The general memoryless InterFerence channel with $K$ sourcedestination pairs $(K$-IFC) considered in this work.

mixed and weak interference, a simple rate splitting in the HK region is optimal to within one bit [18]. The best outer bound may be obtained by intersecting the regions derived by Kramer in [10], by Etkin et al. in [18], and the region independently obtained in [14], [16], [17] and later tighten by Etkin in [19].

Few results are available for more than two users and/or for non-Gaussian channels. Please refer to [20], and references therein, for a more detailed discussion of the past work that we shall only briefly list in the following for sake of space.

General inner bound regions are lacking. A straightforward generalization of the HK approach, whereby each user has a different message for every subset of non-intended receivers, has a super-exponential complexity in the number of users and might be suboptimal in general. In fact, coding schemes that deal directly with the effect of the aggregate interference, rather than with each interferer separately, as with interference alignment [21] and with structured codes [22], are known to achieve a larger number of degrees of freedom than simple HK schemes for the Gaussian noise channel [21], [23], [24]. To the best of the author's knowledge, no outer bounds have been developed for the general (i.e., non-Gaussian) IFC with more than two users.

In Gaussian noise, channels with a special structure have been investigated such as: the "fully symmetric" channel [16], [17], [25], [26], the "cyclic symmetric" channel [27], [28], the three-user channel with "cyclic mixed strong-very strong" interference [29], and the "one-to-many" and the "many-toone" channel [17], [23], [30]. The Degrees of Freedom (DoF) 
of the Gaussian $K$-IFC has received more attention [21], [24], [31]-[33]; the lesson from the DoF analysis is that structured codes appear to outperform purely random codes and that the high-SNR analysis is very sensitive to the way the $K^{2}$ parameters of the $K$-IFC are let grow to infinity.

Of direct relevance for this work are the Gaussian 2IFC outer bounds derived by Kramer in [10, Th.1] and by Etkin et al. in [18], which we seek to generalize to any memoryless channel with any number of users. The basic idea is to give side information to the receiver(s) in such a way that the resulting bound can be single-letterized, does not involve auxiliary random variables and can be computed for channels of interest, such as the Gaussian channel. An extension of [10. Th.1, first proof] to the $K$-user Gaussian channel, with any $K \geq 2$, appeared in [26]; the idea is to provide a group of receivers with sufficient side information so that they can decode a subset of the users as in a Multiple Access Channel (MAC) channel-as also discussed in [25]; the resulting optimization problem however does not appears to have a closed-form solution in general (a closed form result was given in [26] for degraded channels only) and iterative algorithms for its numerical evaluation are discussed in [34]. In this work, we will approach the problem from a different angle: we generalize [10. Th.1, second LMMSE-based proof] rather than [10. Th.1, first "general optimization problem"based proof]. We will show that our bound can be evaluated in closed-form for certain Gaussian channels and it is sumcapacity for some classes of channels. Extensions of [18, Th.1] to the $K$-user Gaussian channel appeared in [17], [35]. In both works, the receivers are given a side information signal that generalizes that of [18, Th.1] whereby entropy terms are related by using the entropy power (EPI) [36] and/or the extremal inequality (EI) [37] rather than chosen so that they cancel one another. In this work we simply generalize the approach of [18. Th.1] to any memoryless channel as it is not obvious what EPI and/or EI are for a general channel.

\section{B. Contributions and Paper Organization}

The main contributions of this work are:

1) In Section II we derive an outer bound on the capacity region of the general memoryless IFC, i.e., not necessarily Gaussian, with an arbitrary number of sourcedestination pairs.

2) In Section III we specialize the bound derived in Section III to the Gaussian channel. In [20], we showed that there exist channel parameters for which our proposed bound is the tightest known for the sum-rate of the Gaussian 3-IFC. Here, we derive the sum-capacity of certain Z-like Gaussian $K$-IFCs. We also discuss how to generalize this sum-capacity result to non-Z Gaussian channels; in particular we offer two alternative proofs for the sum-capacity of the Gaussian degraded channel originally derived in [26].

Section IV concludes the paper. Some of the proofs are in the Appendix.
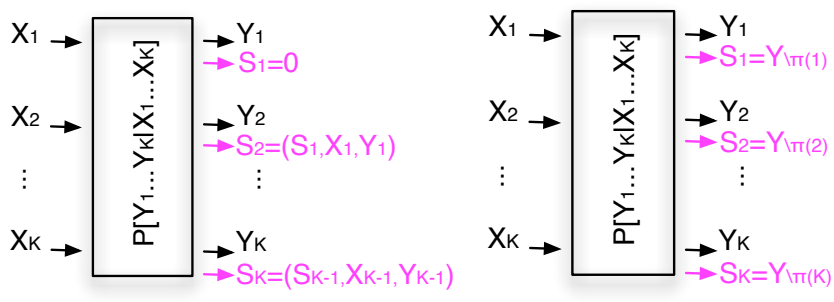

Fig. 2. Receiver side information for the proof of Th 1 Left: for the bound in [1a with $\mathcal{S}=[1: K]$ and $\pi=(1, \ldots, K)$. Right: for the bound in $[1 \mathrm{~b}$.

\section{MAin Result}

Theorem 1. The capacity region of a general memoryless $K$ IFC is contained into:

$\mathcal{O}_{\mathrm{K}-\mathrm{IFC}}=\bigcup_{P: P_{Q}} \prod_{k=1}^{K} P_{X_{k} \mid Q}$
$\sum_{u \in \mathcal{S}} R_{u} \leq \sum_{k=1}^{|\mathcal{S}|}$
$I\left(Y_{\pi_{k}} ; X\left(\left[\pi_{k}: \pi_{|\mathcal{S}|}\right]\right) \mid X\left(\left[\pi_{0}: \pi_{k-1}\right]\right), Y\left(\left[\pi_{0}: \pi_{k-1}\right]\right), X(X) \Pi[\mathcal{S}]\right.$
$\left.\left.\mathcal{S}^{c}\right), Q\right)$,

$$
\left.\sum_{u \in \mathcal{S}} R_{u} \leq \sum_{k=1}^{|\mathcal{S}|} H\left(Y_{k} \mid Y_{\backslash \pi_{k}}\right)-H\left(Y_{\backslash \pi_{k}} \mid Y_{k}, X_{1}, \ldots, X_{K}\right),\right\},
$$

where the union is over all input distributions $P=$ $P_{X_{1}, \ldots, X_{K}, Q}$ that factorize as $P_{Q} \prod_{k=1}^{K} P_{X_{k} \mid Q}$ and the intersection is over all subsets $\mathcal{S}$ of the user-index set $[1: K]$ and over all permutations $\pi$ of the elements of $\mathcal{S}$. The random variable $Y_{\backslash k}$ has the same distribution of $\left.Y_{k}\right|_{X_{k}}$ (i.e., the conditional distribution of an output given its intended input). A random variable with subscript $\pi_{0}$ is a constant.

Proof: The details of the proof may be found in the Appendix. The key idea is to provide the $k$-th receiver, $k \in[1: K]$, with the side information $S_{k}$ shown in Fig. 2.

We remark that:

1) Th 1 holds for any memoryless IFC and for any number of users.

2) Since the capacity region of a $K$-user IFC does not depend on the joint transition probability $P_{Y_{1}, \ldots, Y_{K} \mid X_{1}, \ldots, X_{K}}$ (because the receivers cannot cooperate), but only on the marginal transition probabilities $P_{Y_{k} \mid X_{1}, \ldots, X_{K}}, k \in[1: K]$, each bound in Th. 1 (one for each pair $(\mathcal{S}, \pi)$ ) can be optimized with respect to the joint probability $P_{Y_{1}, \ldots, Y_{K} \mid X_{1}, \ldots, X_{K}}$ as long as the marginal probabilities are preserved.

3) The bound in (1a) reduces to [10, Th.1] for the Gaussian 2-IFC when $\mathcal{X}_{3}=\emptyset$ (see [10, eq.(34)] which inspired the side information structure given on the left side of Fig. 2).

4) The bound in (1b) reduces to [18, Th.1] for the Gaussian 2-IFC when $\mathcal{X}_{3}=\emptyset$ by setting $S_{1}=Y_{\backslash 2}$ and $S_{2}=Y_{\backslash 1}$. 
The bound in $[1 \mathrm{~b}]$ is tighter than [18, Th.1] because the correlation coefficient between the Gaussian noise of the channel output $Y_{k}$ and the Gaussian noise of the side information $Y_{\backslash \pi_{k}},\left(k, \pi_{k}\right) \in[1: K]^{2}$, can be optimized so as to get the tightest bound. It is however not tighter than the bound independently obtained in [14], [16], [17] for the 2-user Gaussian channel.

5) From 1a we get $N(K)=\sum_{k=1}^{K}\left(\begin{array}{c}K \\ k\end{array}\right) k$ ! rate bounds. For $K=2$, the $N(2)=4$ bounds are as in [10, Th.1] (two single-rate bounds and two sum-rate bounds). For $K \geq 3$, the $N(K)-K^{2}$ bounds that involve at least three rates cannot be simply derived by silencing all but two users and then by applying [10, Th.1] to the resulting 2-IFC. The number of bounds grows exponentially with $K: N(3)=15, N(4)=52, N(5)=325$, etc.

Similarly, from $(1 \mathrm{~b})$ we get $N(K)$ bounds; those that involve at least three rates cannot be obtained by simply applying the 2-IFC sum-rate bound in [18, Th.1].

6) $\mathrm{Th} 1$ can be easily evaluated. For example, the "Gaussian maximizes entropy" [36] suffices to guarantee that a jointly Gaussian input is optimal for Gaussian channels.

7) $\mathrm{Th} 1$ can be extended to other memoryless channels without receiver cooperation. For example, the 2-user cognitive channel was considered in [38], the 2-IFC with a cognitive relay in [39], and the 2-IFC with generalized feedback (a.k.a. source cooperation) in [40]-[42].

For the $K$-IFC with generalized feedback for example, Th11 must be modified as follows: (a) replace each channel output $Y_{k}$ with the pair $\left(Y_{k}, Y_{\mathrm{GF}, k}\right)$, where $Y_{\mathrm{GF}, k}$ is the channel output observed at transmitter $k, k \in[1: K]$, (b) consider the union over all possible joint input distributions $P_{X_{1}, \ldots, X_{K}}$ (because the generalized feedback enables source cooperation which results in correlated inputs); (c) choose the worst joint transition probability $P_{Y_{1}, \ldots, Y_{K} \mid X_{1}, \ldots, X_{K}, Y_{\mathrm{GF}, 1}, \ldots, Y_{\mathrm{GF}, K}}$ that preserves the marginals $P_{Y_{k} \mid X_{1}, \ldots, X_{K}, Y_{\mathrm{GF}, 1}, \ldots, Y_{\mathrm{GF}, K}}$, $k \in[1: K]$.

Similar extensions are possible for other channels.

8) For the Gaussian 2-IFC, besides the bounds in [10] and in [18] that we generalized in $\mathrm{Th}$, the following outer bounds are known: [10, Th.2] [14], [16], [17] and [19]. These bounds are tighter than [18, Th.1] for some weak interference parameters. It is left for future work to generalize these 2-user Gaussian channel bounds to non-Gaussian channels with more than two users. We note that the common feature of these bounds is to generalize the class of genie signals of [18, Th.1] by relating entropy terms rather than canceling them (see proof of $\mathrm{Th}, 1$; this is done by using the entropy power (EPI) [36] and/or the extremal inequality (EI) [37]; the extension of the EPI and/or the EI to general (i.e., nonGaussian) channels is not trivial.

\section{GAUSSIAN CHANNELS}

In this section we first introduce the Gaussian channel model (subsection III-A). We then show that Th1 eq. (1a) gives the sum-capacity for certain Z-channels (subsection III-B). We conclude with Subsection III-C where we discuss how to extend the result of Subsection III-B to non-Z channels; in doing so we show that $\mathrm{Th} 1$ eq. (1a) gives the sum-rate capacity of degraded channels, thereby providing an alternative proof for the result of [26]; we also offer an alternate proof for the sum-rate capacity of the degraded Gaussian $K$-IFC by generalizing an argument originally devised by Sato for the degraded Gaussian 2-IFC [8].

\section{A. The Gaussian Channel Model}

A SISO (single input single output) complex-valued Gaussian $K$-IFC in standard form has outputs:

$$
Y_{i}=\sum_{k=1}^{K} h_{i, k} X_{k}+Z_{i}
$$

with input power constraint $\mathbb{E}\left[\left|X_{i}\right|^{2}\right] \leq 1$ and noise $Z_{i} \sim$ $\mathcal{N}(0,1), i \in[1: K]$. The correlation among the Gaussian noises is irrelevant since the capacity only depends on the marginal noise distributions. The channel gains are fixed and are known to all terminals. Without loss of generality, the direct link gains $h_{i, i}, i \in[1: K]$, can be taken to be real-valued (because receiver $i$ can compensate for the phase of $h_{i, i}$ ) and strictly positive (if $\left|h_{i, i}\right|^{2}=0$ then the SNR at receiver $i$ is zero even in absence of interference, which implies that $R_{i}=0$ is optimal, i.e., the system has effectively one less user). The Gaussian $K$-IFC is completely specified by the channel matrix $\boldsymbol{H}:[\boldsymbol{H}]_{i, j}=h_{i, j},(i, j) \in[1: K] \times[1: K]$.

In the following we adopted the Matlab-like convention that $\boldsymbol{H}_{\mathcal{R}, \mathcal{C}}$ in the $|\mathcal{R}| \times|\mathcal{C}|$ matrix obtained from $\boldsymbol{H}$ by retaining the rows indexed by $\mathcal{R}$ and the columns indexed by $\mathcal{C}$.

\section{B. Sum-capacity of Z-like channels}

Here we consider a class of Gaussian $K$-IFCs for which the channel matrix $\boldsymbol{H}$ is upper triangular. This class of channels can be thought of as the multi-user generalization of the 2IFC Z-channel [15]. The following theorem establishes the sum-capacity for a subset of Z-channels for which treating interference as noise is optimal:

Theorem 2. Consider a $K \times K$ noise covariance matrix $\boldsymbol{\Sigma}_{K}$ defined recursively as follows: let $\boldsymbol{\Sigma}_{1}=[1]$ and $\forall k=$ $2, \ldots, K$ let

$\boldsymbol{\Sigma}_{k}=\left(\begin{array}{cc}\boldsymbol{\Sigma}_{k-1} & \boldsymbol{\rho}_{k-1} \\ \boldsymbol{\rho}_{k-1}^{H} & 1\end{array}\right), \boldsymbol{\rho}_{k-1} \in \mathbb{C}^{k-1}: \boldsymbol{\rho}_{k-1} \boldsymbol{\rho}_{k-1}^{H} \preceq \boldsymbol{\Sigma}_{1: k-1}$.

Consider a channel matrix $\boldsymbol{H}$ whose upper triangular part is defined recursively as follows: for $k=K, \ldots, 2$

$$
\begin{aligned}
& \boldsymbol{H}_{[1: k-1],[k]}=\frac{\boldsymbol{H}_{[k],[k]}}{1+\left\|\boldsymbol{H}_{[k],[k+1 ; K]}\right\|^{2}} \\
&\left(\boldsymbol{\rho}_{k-1}+\boldsymbol{H}_{[1: k-1],[k+1: K]} \boldsymbol{H}_{[k],[k+1: K]}\right),
\end{aligned}
$$

while the entries below the main diagonal of $\boldsymbol{H}$ are zero. For the channel defined by (2), the sum-rate capacity is given 
by (1a) and equals:

$$
\sum_{k=1}^{K} R_{k} \leq \sum_{k=1}^{K} \log \left(1+\frac{\left|h_{k, k}\right|^{2}}{1+\sum_{i=k+1}^{K}\left|h_{k, i}\right|^{2}}\right)
$$

Proof: Since every mutual information term in (1a) contains all the inputs, the "Gaussian maximizes entropy" principle [36] assures that iid $\mathcal{N}(0,1)$ inputs are optimal. Consider $\mathcal{S}=[1: K]$ with $\pi=(1, \ldots, K)$ in $(1 \mathrm{a})$ and rewrite the sum-rate as:

$$
\begin{aligned}
& \sum_{k=1}^{K} R_{k} \leq \min _{\boldsymbol{\Sigma}_{K}}\left\{\sum_{k=1}^{K} I_{k}\left(\boldsymbol{\Sigma}_{k}\right)\right\} \\
& I_{k}\left(\boldsymbol{\Sigma}_{k}\right) \triangleq I\left(Y_{1}, \ldots, Y_{k-1}, Y_{k} ; X_{k} \mid X_{1}, \ldots, X_{k-1}\right)
\end{aligned}
$$

The channel matrix $\boldsymbol{H}$ defined by (2) is such that for each $k=K, \ldots, 2$ :

$$
\begin{aligned}
& \mathbb{E}\left[Y_{1}, \ldots, Y_{k-1} \mid Y_{k}, X_{1}, \ldots, X_{k-1}, X_{k}\right] \\
= & \mathbb{E}\left[Y_{1}, \ldots, Y_{k-1} \mid Y_{k}, X_{1}, \ldots, X_{k-1}\right],
\end{aligned}
$$

that is, conditioned on $\left(X_{1}, \ldots, X_{k-1}\right)$ the set of outputs $\left(Y_{1}, \ldots, Y_{k-1}\right)$ is a degraded version of $Y_{k}$ and thus:

$$
\begin{aligned}
I_{k}\left(\boldsymbol{\Sigma}_{k}\right) & =I\left(Y_{k} ; X_{k} \mid X_{1}, \ldots, X_{k-1}\right) \\
& =\log \left(1+\frac{\left|h_{k, k}\right|^{2}}{1+\sum_{i=k+1}^{K}\left|h_{k, i}\right|^{2}}\right) \triangleq r_{k} .
\end{aligned}
$$

By summing the rates in $[6$ over all $k \in[1: K]$ we obtain the sum-rate upper bound in (3). The upper bound in (3) can be achieved by simply treating interference as noise at each receiver (recall that for the Z-channel, the $k$-th receiver is interfered by $\left(X_{k+1}, \ldots, X_{K}\right)$ only).

By considering all possible covariance matrices $\Sigma_{K}, \operatorname{Th} 2$ identifies a novel class of channels for which treating interference as noise is sum-rate optimal (besides those in [17, Th.4, Th.5, and Th.7] and [35. Th.3]) as shown in the following examples. The correspondence between channel matrices and noise covariance matrices given by (2) is interesting in itself and deserves further analysis.

Example 1. Th 2 assures that treating interference as noise is optimal for all channels that can be built as in (2) from a covariance matrix of the type:

$$
\boldsymbol{\Sigma}_{K}=\left(\begin{array}{cccc}
1 & v_{2} & \ldots & v_{K} \\
v_{2}^{*} & & & \\
\vdots & & \boldsymbol{I}_{k-1} & \\
v_{K}^{*} & & &
\end{array}\right): \sum_{k=2}^{K}\left|v_{k}\right|^{2} \leq 1 .
$$

The resulting channel has gains $h_{1, k}=v_{k} h_{k, k}, k=2, \ldots, K$ and zero for the remaining non-diagonal entries; this channel is to the so-called many-to-one channel [23]. The condition $\sum_{k=2}^{K}\left|v_{k}\right|^{2} \leq 1$ identifies a subset of many-to-one channels for which treating interference as noise is optimal. The condition $\sum_{k=2}^{K}\left|v_{k}\right|^{2} \leq 1$ is equivalent to [17, Th.4], thus $\operatorname{Th} 2$ generalizes [17, Th.4].

The relationship between the class of channels identified by Th 2 and that identified by [35, Th.3] (of which [17, Th.4 and
Th.5] are special cases) is subject of current investigation. We note that [35, Th.3] is obtained from a generalization of [18. Th.1] while Th 2 from a generalization of [10, Th.1], it is thus possible that [35, Th.3] and $\mathrm{Th} 2$ do not imply one another.

Example 2. Consider channels that can be obtained as in (2) from a rank-one covariance matrix of the type:

$$
\boldsymbol{\Sigma}_{K}:\left[\boldsymbol{\Sigma}_{K}\right]_{i, j}=a_{i} / a_{j}
$$

for some $\left(a_{1}, \ldots, a_{K}\right) \in \mathbb{C}^{K}$. The resulting channel has entries $h_{i, j}=h_{j, j} a_{i} / a_{j}, j \in[i: K]$ and $i \in[1: K]$, and zero for the remaining non-diagonal entries. For these channels the sum-capacity is:

$$
\sum_{k=1}^{K} \log \left(1+\frac{\left|a_{k}\right|^{2}\left|h_{k, k} / a_{k}\right|^{2}}{1+\left|a_{k}\right|^{2} \sum_{j=k+1}^{K}\left|h_{j, j} / a_{j}\right|^{2}}\right) .
$$

In the next subsection we relate the channels considered in Example 2 with the class of degraded channels studied in [26].

\section{Sum-capacity of non-Z channels}

The condition expressed by (2) is only sufficient for the achievability of (3). In general, the expression in (3) is an upper bound to the sum-capacity of channels for which the upper triangular part of $\boldsymbol{H}$ can be expressed as in (2) and the entries below the main diagonal have any arbitrary value. For such channels, the entries below the main diagonal might need to satisfy some extra constraints (besides (2)) in order for (3) to be achievable. The following discusses an example of such extra constraints.

The expression in (3) suggests the following achievable strategy for non- $Z$ channels: the $k$-th receiver first decodes users $1, \ldots, k-1$, then strip them from its received signal, and finally decodes its intended message by treating the signal of users $k+1, \ldots, K$ as noise; with this "successive decoding strategy" the rate-tuplet $\left(r_{1}, \ldots, r_{K}\right)$ in 6 is achievable if:

Theorem 3. The sum-rate in (3) is achievable for a channel $\boldsymbol{H}$ such that the upper triangular part of $\boldsymbol{H}$ can be expressed as in (2) and such that the entries below the main diagonal satisfy:

$$
\begin{aligned}
& \left(r_{1}, \ldots, r_{K}\right) \in \bigcap_{k \in[1: K]} \bigcap_{\mathcal{S}_{k} \subseteq[1: k-1]}\left\{\left(R_{1}, \ldots, R_{K}\right) \in \mathbb{R}_{+}^{K}:\right. \\
& \left.R_{k}+\sum_{j \in \mathcal{S}_{k}} R_{j} \leq \log \left(1+\frac{\left|h_{k, k}\right|^{2}+\sum_{j \in \mathcal{S}_{k}}\left|h_{k, j}\right|^{2}}{1+\sum_{i=k+1}^{K}\left|h_{k, i}\right|^{2}}\right)\right\} .
\end{aligned}
$$

for $\left(r_{1}, \ldots, r_{K}\right)$ defined in (6).

Proof: The proof follows from the previous discussion. The achievable region in $\mathrm{Th} 3$ is the intersection of $K \mathrm{MAC}$ regions where only the constraints that involve the intended rate have been retained.

As a corollary to $\mathrm{Th} 3$ we have:

Corollary 4. Degraded channels, that is, channels for which $\boldsymbol{H}$ has rank one, satisfy the assumptions of $T h 3$ 
Proof: Consider a $K$-IFC with unit rank channel matrix $\boldsymbol{H}=\boldsymbol{a} \boldsymbol{b}^{H}$, for some $K$-length column vectors $\boldsymbol{a}$ and $\boldsymbol{b}$ (notice that these channels have upper triangular part as in Example 2 with $\left.b_{k}^{*}=h_{k, k} / a_{k}\right)$. Without loss of generality, assume that the entries of the vector $\boldsymbol{a}$ satisfy $\left|a_{1}\right| \leq\left|a_{2}\right| \ldots \leq\left|a_{K}\right|$. With this ordering the channel outputs from a Markov chain:

$$
\begin{aligned}
& X_{\mathrm{eq}} \triangleq \sum_{k=1}^{X} X_{k} b_{k}^{*} \rightarrow Y_{K} \rightarrow Y_{K-1} \ldots \rightarrow Y_{1}, \\
& Y_{k} \sim a_{k} X_{\mathrm{eq}}+Z_{k}, k \in[1: K] .
\end{aligned}
$$

For this channel, clearly the $k$-th decoder can decode all users with index $i<k$ without imposing any rate penalty to these users; thus sum-rate in (3) is achievable.

Corollary 4 offers a simple proof for the sum-capacity result of [26]; this implies that Th 3 generalizes the result of [26].

Another proof for the converse part of Corollary 4 can be obtained by generalizing the bound for the degraded Gaussian 2-IFC proposed by Sato in [1]. We have:

Theorem 5. The capacity of the Gaussian K-IFC with channel matrix $\boldsymbol{H}=\boldsymbol{a} \boldsymbol{b}^{H}$, such that $\left|a_{1}\right| \leq\left|a_{2}\right| \ldots \leq\left|a_{K}\right|$, is outer bounded by:

$$
R_{k} \leq \log \left(1+\frac{\beta_{u}\|\boldsymbol{b}\|^{2}\left|a_{k}\right|^{2}}{1+\left(\sum_{j=k+1}^{K} \beta_{j}\|\boldsymbol{b}\|^{2}\right)\left|a_{k}\right|^{2}}\right),
$$

for all $\beta_{k} \geq 0, k \in[1: K]$, such that $\sum_{k=1}^{K} \beta_{k}=1$.

Proof: By letting the transmitters cooperate, the capacity of the unit-rank $K$-IFC is outer bounded by the capacity of a $K$-user degraded SISO Broadcast Channel (BC) with input $X_{\text {eq }}=\sum_{k=1}^{K} b_{k}^{*} X_{k}$, input power constraint $\mathbb{E}\left[\left.X_{\text {eq }}\right|^{2}\right] \leq\|\boldsymbol{b}\|^{2}$, and outputs $Y_{k}=a_{k} X_{\mathrm{eq}}+Z_{k}, k \in[1: K]$. The capacity of this degraded $K$-user $\mathrm{BC}$ is given by [8] [36].

By using the outer bound in $\mathrm{Th} 5$ we have the following very simple proof for the converse part of Corollary 4 , by letting $\beta_{u}\|\boldsymbol{b}\|^{2}=\left|b_{k}\right|^{2}$ in $(8)$ we immediately obtain the upper bound in (3), which is equivalent to (7) with $b_{k}^{*}=h_{k, k} / a_{k}$.

\section{CONClusions}

In this work we developed a framework to derive an outer bound for the general memoryless interference channel with an arbitrary number of source-destination pairs. For the Gaussian channel, we showed that the proposed bound gives the sumcapacity for certain channels, including some Z-channels and degraded channels.

\section{APPENDIX}

Proof of [1a). Consider a non-empty subset $\mathcal{S}$ of $[1: K]$ and let $\mathcal{S}^{c}$ be its complement in $[1: K]$. Consider without loss of generality the permutation $\pi=(1,2, \ldots,|S|)$ (the others are obtained by relabeling the users). We use the following conventions: for a set $\mathcal{S}, W(\mathcal{S})=\left\{W_{i}: i \in \mathcal{S}\right\}$. We have:

$$
\begin{aligned}
& n \sum_{k=1}^{|\mathcal{S}|}\left(R_{k}-\epsilon_{n}\right) \stackrel{(\mathrm{a})}{=} \sum_{k=1}^{|\mathcal{S}|} I\left(X_{k}^{n} ; Y_{k}^{n}\right) \\
& \stackrel{(\mathrm{b})}{\leq} \sum_{k=1}^{|\mathcal{S}|} I\left(X_{k}^{n} ; Y_{k}^{n}, Y_{1}^{n}, \ldots, Y_{k-1}^{n}, X_{1}^{n}, \ldots, X_{k-1}^{n}, X^{n}\left(\mathcal{S}^{c}\right)\right) \\
& \stackrel{(\mathrm{c})}{=} \sum_{k=1}^{|\mathcal{S}|} I\left(X_{k}^{n} ; Y_{k}^{n}, Y_{1}^{n}, \ldots, Y_{k-1}^{n} \mid X_{1}^{n}, \ldots, X_{k-1}^{n}, X^{n}\left(\mathcal{S}^{c}\right)\right) \\
& \stackrel{(\mathrm{d})}{=} \sum_{k=1}^{|\mathcal{S}|} \sum_{\ell=1}^{k} I\left(X_{k}^{n} ; Y_{\ell}^{n}, \mid X_{1}^{n}, \ldots, X_{k-1}^{n}, X^{n}\left(\mathcal{S}^{c}\right), Y_{1}^{n}, \ldots, Y_{\ell-1}^{n}\right) \\
& \stackrel{(\mathrm{e})}{=} \sum_{\ell=1}^{|\mathcal{S}|} \sum_{k=\ell}^{|\mathcal{S}|} I\left(X_{k}^{n} ; Y_{\ell}^{n}, \mid X_{1}^{n}, Y_{1}^{n}, \ldots, X_{\ell-1}^{n}, Y_{\ell-1}^{n}, X^{n}\left(\mathcal{S}^{c}\right),\right. \\
& \stackrel{(\mathrm{f})}{=} \sum_{\ell=1}^{|\mathcal{S}|} I\left(X_{\ell}^{n}, \ldots, X_{|\mathcal{S}|}^{n} ; Y_{\ell}^{n}, \mid X_{1}^{n}, Y_{1}^{n}, \ldots, X_{\ell-1}^{n}\right) \\
& \stackrel{(\mathrm{g})}{\leq} n \sum_{\ell=1}^{|\mathcal{S}|} I\left(X_{\ell}, \ldots, X_{|\mathcal{S}|}^{n} ; Y_{\ell} \mid X_{1}, Y_{1}, \ldots, X_{\ell-1}, Y_{\ell-1}, X\left(\mathcal{S}^{c}\right)\right) \\
&
\end{aligned}
$$

where the different (in)equalities follow from: (a) Fano's inequality, (b) non-negativity of mutual information (i.e., add side information at the receivers as in Fig. 2), (c) independence of messages (and thus of codewords), (d) chain rule for mutual information, (e) swap order of summation, (f) chain rule for mutual information, (g) "conditioning reduces entropy", memoryless property of the channel, and by introducing a "time sharing" random variable $Q$ uniformly distributed on $[1: n]$ and independent of everything else.

Proof of 1b]. Consider a non-empty subset $\mathcal{S}$ of $[1: K]$,

$$
\begin{aligned}
& \sum_{k \in \mathcal{S}} n\left(R_{k}-\epsilon_{n}\right) \leq \sum_{k \in \mathcal{S}} I\left(X_{k}^{n} ; Y_{k}^{n}\right) \\
& \leq \sum_{k \in \mathcal{S}} I\left(X_{k}^{n} ; Y_{k}^{n}, S_{k}^{n}\right) \\
& =\sum_{k \in \mathcal{S}} H\left(S_{k}^{n}\right)+H\left(Y_{k}^{n} \mid S_{k}^{n}\right)-H\left(Y_{k}^{n} \mid X_{k}^{n}\right)-H\left(S_{k}^{n} \mid X_{k}^{n}, Y_{k}^{n}\right) \\
& \stackrel{(\text { a) }}{\leq} \sum_{k \in \mathcal{S}} H\left(Y_{k}^{n} \mid S_{k}^{n}\right)-H\left(S_{k}^{n} \mid Y_{k}^{n}, X_{1}^{n}, \ldots, X_{K}^{n}\right),
\end{aligned}
$$

where the inequality in (a) requires:

$\sum_{k \in \mathcal{S}}\left(H\left(S_{k}^{n}\right)-H\left(Y_{k}^{n} \mid X_{k}^{n}\right)-I\left(S_{k}^{n} ; X_{1}^{n}, \ldots, X_{K}^{n} \mid X_{k}^{n}, Y_{k}^{n}\right)\right) \leq 0$.

A good candidate for the side information is as in Fig. 2 inspired by [18]

$$
\left\{S_{k}, k \in \mathcal{S}\right\}=\left\{Y_{\backslash k}, k \in \mathcal{S}\right\}, \quad \forall \mathcal{S} \subseteq[1: K],
$$

where we defined $\left.Y_{\backslash k} \sim Y_{k}\right|_{X_{k}}$. 


\section{ACKNOWLEDGMENT}

This work was partially funded by NSF under award number 0643954. The contents of this article are solely the responsibility of the authors and do not necessarily represent the official views of the NSF.

\section{REFERENCES}

[1] H. Sato, "On the capacity region of a discrete two-user channel for strong interference," in IEEE Trans. Inform. Theory, vol. 24(3), May 1978, pp. 377-379.

[2] M. H. M. Costa and A. A. E. Gamal, "The capacity region of the discrete memoryless interference channel with strong interference," in IEEE Trans. Inform. Theory, vol. 33(5), Sept 1987, pp. 710-711.

[3] A. B. Carleial, "A case where interference does not reduce capacity," in IEEE Trans. Inform. Theory, vol. 21(5), Sept 1975, pp. 569-570.

[4] A. A. E. Gamal and M. H. M. Costa, "The capacity region of a class of deterministic interference channels," in IEEE Trans. Inform. Theory, vol. 28(2), March 1982, pp. 343-346.

[5] N. Liu and S. Ulukus, "The capacity region of a class of discrete degraded interference channels," in IEEE Trans. Inform. Theory, vol. 54(9), Sept 2008, pp. 4372-4378.

[6] R. Benzel, "The capacity region of a class of discrete additive degraded interference channels," IEEE Trans. Inform. Theory, vol. IT-25, pp. 228 231, Mar 1979.

[7] T. S. Han and K. Kobayashi, "A new achievable rate region for the interference channel," in IEEE Trans. Inform. Theory, vol. 27(1), Jan 1981, pp. $49-60$.

[8] H. Sato, "Two-user communication channels," in IEEE Trans. Inform. Theory, vol. 23(3), 1977, pp. 295 - 304.

[9] A. B. Carleial, "Outer bounds on the capacity of interference channels," in IEEE Trans. Inform. Theory, vol. 29, no. 4, July 1983, pp. 602-606.

[10] G. Kramer, "Outer bounds on the capacity of gaussian interference channels," in IEEE Trans. Inform. Theory, vol. 50, no. 3, Jan 2004, pp. $581-586$.

[11] H. Sato, "The capacity of the gaussian interference channel under strong interference," in IEEE Trans. Inform. Theory, vol. 27(6), Nov 1981, pp. 786-788.

[12] M. H. M. Costa, "On the gaussian interference channel," in IEEE Trans. Inform. Theory, vol. 31(5), Sept 1985, pp. 607-615.

[13] D. Tuninetti and Y. Weng, "On gaussian mixed interference channels," in IEEE International Symposium Information Theory, Toronto, Canada, July 2008 .

[14] A. S. Motahari and A. K. Khandani, "Capacity bounds for the gaussian interference channel," IEEE Trans. Inform. Theory, vol. 55, no. 2, pp. 620-643, 2009.

[15] I. Sason, "On achievable rate regions for the gaussian interference channel," in IEEE Trans. Inform. Theory, vol. 50(6), June 2004, pp. $1345-1356$.

[16] X. Shang, G. Kramer, and B. Chen;, "A new outer bound and the noisyinterference sumrate capacity for gaussian interference channels," in IEEE Trans. Inform. Theory, vol. 55(2), Feb 2009, pp. 689 - 699.

[17] V. S. Annapureddy and V. V. Veeravalli, "Gaussian interference networks: Sum capacity in the low-interference regime and new outer bounds on the capacity region," in IEEE Trans. Inform. Theory, vol. 55(7), July 2009, pp. $3032-3050$.

[18] R. H. Etkin, D. N. C. Tse, and H. Wang, "Gaussian interference channel capacity to within one bit," IEEE Trans. Inform. Theory, vol. 54, no. 12, pp. $5534-5562,2008$

[19] R. Etkin, "New sum-rate upper bound for the two-user gaussian interference channel," Information Theory, 2009. ISIT 2009. IEEE International Symposium on, pp. 2582 - 2586, 2009.

[20] D. Tuninetti, "A new sum-rate outer bound for interference channels with three source-destination pairs," Proceedings of the Information Theory and Applications Workshop, 2011, San Diego, CA USA, Feb 2011.

[21] V. R. Cadambe and S. A. Jafar, "Interference alignment and the degrees of freedom for the $\mathrm{k}$ user interference channel," IEEE Trans. Inform. Theory, vol. 54, no. 8, pp. 3425-3441, Aug 2008.

[22] B. Nazer and M. Gastpar, "Computation over multiple-access channels," IEEE Trans. Inform. Theory, vol. 53, no. 10, pp. 3498-3516, Oct. 2007.

[23] G. Bresler, A. Parekh, , and D. Tse, "The approximate capacity of the many-to-one and one-to-many gaussian interference channel, arxiv:0809.3554," Proceedings of the Allerton Conference, Sept 2007.
[24] S. Sridharan, A. Jafarian, S. Vishwanath, S. Jafar, and S. Shamai, "A layered lattice coding scheme for a class of three user gaussan interference channels," Communication, Control, and Computing, 2008 46th Annual Allerton Conference on, pp. 531 - 538, Sept 2008.

[25] V. R. Cadambe and S. A. Jafar, "Parallel gaussian interference channels are not always separable," IEEE Trans. Inform. Theory, vol. 55(9), pp. 3983-3990, Sept 2009

[26] J. Jose and S. Vishwanath, "Sum capacity of k user gaussian degraded interference channels," Proc. IEEE Info. Theory Workshop (ITW), Dublin, Ireland., Sept 2010.

[27] L. Zhou and W. Yu, "On the symmetric capacity of the k-user symmetric cyclic gaussian interference channel," Proceedings of Conference on Information Science and Systems (CISS), Princeton, NJ, March 2010.

[28] B. Bandemer, G. Vazquez-Vilar, and A. E. Gamal, "On the sum capacity of a class of cyclically symmetric deterministic interference channels," Proc. IEEE International Symposium on Information Theory, Seoul, Korea, p. 26222626, July 2009.

[29] A. Chaaban and A. Sezgin, "The capacity region of the 3-user gaussian interference channel with mixed strong-very strong interference, arxiv:1010.4911," Submitted to ISIT 2011, 2010.

[30] J. A., H. Wang, and V. P., "On network interference management," pp. $307-312$, Sept 2007.

[31] S. Sridharan, A. Jafarian, S. Vishwanath, and S. A. Jafar, "Capacity of symmetric k-user gaussian very strong interference channels," Globecom 2008,2008

[32] S. A. Jafar and S. Vishwanath, "Generalized degrees of freedom of the symmetric gaussian k user interference channel," IEEE Trans. Inform. Theory, vol. 56(7), pp. 3297-3303, July 2010.

[33] R. Etkin and E. Ordentlich, "On the degrees-of-freedom of the k-user gaussian interference channel," Information Theory, 2009. ISIT 2009. IEEE International Symposium on, pp. 1919 - 1923, 2009.

[34] J. Jose, N. Prasad, M. Khojastepour, and S. Rangarajan, "On robust weighted-sum rate maximization in mimo interference networks," Proc. IEEE ICC 2011, Kyoto, Japan, June 2011.

[35] X. Shang, G. Kramer, and B. Chen, "New outer bounds on the capacity of gaussian interference networks," in IEEE Int. Symp. Inform. Theory, Toronto, Canada, July 2008, pp. $245-249$.

[36] T. Cover and J. Thomas, Elements of information theory. New York: Wiley, 1991.

[37] T. Liu and P. Viswanath, "An extremal inequality motivated by multiterminal information theoretic problems," IEEE Trans. Inform. Theory, vol. 53, no. 5, pp. 1839 - 1851, 2007.

[38] S. Rini, D. Tuninetti, and N. Devroye, "New inner and outer bounds for the discrete memoryless cognitive channel and some capacity results," Information Theory, IEEE Transactions on, 2010, in print, preprint at arXiv: 1003.4328 .

[39] - "Outer bounds for the interference channel with a cognitive relay," Proc. IEEE Information Theory Workshop (ITW), Dublin, Ireland (preprint at arXiv:1004.4944), Sep 2010.

[40] S. Yang and D. Tuninetti, "A new sum-rate outer bound for gaussian interference channels with generalized feedback," in Proceedings of 2009 IEEE International Symposium on Information Theory (ISIT 2009), Seoul, South Korea, June 2009.

[41] D. Tuninetti, "An outer bound region for interference channels with generalized feedback," Proceedings of the Information Theory and Applications Workshop, 2010, San Diego, CA USA, Jan 2010.

[42] V. Prabhakaran and P. Viswanath, "Interference channels with source cooperation," submitted to IEEE Trans. Info. Theory in May 2009, Arxiv preprint arXiv:0905.3109v1, 2009. 